\title{
Erosion Mechanism and Sensitivity Parameter Analysis of an Innovative Shaped Curved Pipeline
}

\author{
Li MO*, Zhiyuan WANG**, Shulu FENG***, Jiadai DU****, Hao YI*****, Jiahui TANG****** \\ *School of Mechatronic Engineering, Southwest Petroleum University, Chengdu 610500, China; \\ E-mail: 315812917@qq.com \\ **National Joint Engineering Research Center for Abrasion Control and Molding of Metal Materials, Luoyang 471003, \\ China; E-mail: 515008353@qq.com \\ ***PetroChina Southwest Pipeline Branch Company, Chengdu 610094, China; E-mail: fengshulu@petrochina.com.cn \\ ****School of Mechatronic Engineering, Southwest Petroleum University, Chengdu 610500, China; \\ E-mail:2845411253@qq.com \\ *****School of Mechatronic Engineering, Southwest Petroleum University, Chengdu 610500, China; \\ E-mail: 1657670445@qq.com \\ ******School of Mechatronic Engineering, Southwest Petroleum University, Chengdu 610500, China; \\ E-mail:2876464639@qq.com
}

cross $^{\text {ref }}$ http://dx.doi.org/10.5755/j01.mech.26.6.24767

\section{Introduction}

In the process of natural gas transportation, there are some small solid particles, which will cause erosion pipelines. Especially for elbows with complex internal flow field, the erosion of elbows is about 50 times more serious than that of straight sections [1]. Therefore, it is of great significance to study reasonable elbow structure to reduce the erosion of elbows to ensure the safety of natural gas transportation.

Particle erosion phenomenon is very complex. The factors affecting the severity of erosion include fluid velocity, particle rate, fluid properties, particle diameter, pipe material, and geometric characteristic size and shape. In recent years, due to the continuous development of computational fluid dynamics (CFD), discrete particle model (DPM) has been widely used to predict the erosion of solid particles in fluids. Finnie [2, 3] first proposed a micro-geometric model of plastic materials. He believed that micro-cutting was the cause of erosion of plastic materials, and proposed that strengthening metal surface strength could reduce erosion wear rate. Salik J [4] et al. found that particle shape has a great influence on erosion wear. McLaury [5] proposed a generalized erosion prediction program, which consists of three steps: flow simulation, particle tracking and erosion prediction, which are the main steps of many erosion models. Chen et al. [6] proposed a two-phase flow erosion prediction model based on CFD discrete element method (DEM) for elbow connections. Therefore, the erosion wear of elbow can be predicted effectively by using computational fluid dynamics (CFD) method.

At present, many scholars begin to consider reducing the erosion wear of elbow from the aspect of elbow structure. Wenshan Peng and Xuewen Cao used CFD model to solve the liquid-solid flow in the pipe bend. It produces results that are closest to the experimental data [7, 8]. Santos et al. reported the insertion of a twisted tape upstream of a bend could attenuate the direct collisions against the same spot in the bend wall because of the swirl imparted by the tape [9]. S.Laín and M.Sommerfeld [10]study the Euler/Lagrange approach in combination with a proper turbulence model and full two-way coupling is applied for erosion estimation due to particle conveying along a horizontal to vertical pipe bend. The results show that an increase in wall roughness decreases remarkably the penetration ratio and also changes the shape of the erosion pattern, eliminating the two antenna-like zones obtained for smooth wall computations. Duarte, C. A. R. \& de Souza, F. J. [11] propose a novel pipe wall design in order to reduce the erosion on a $90^{\circ}$ elbow. The simulations show that a reduction of erosion peak up to $33 \%$ can be achieved in a pipeline equipped with the twisted pipe wall. Hongjun Zhu and Shuai Li [12] study a trapezoidal rib installed at different positions on the extrados of a $90^{\circ}$ elbow is investigated numerically. The results show that $\mathrm{A}$ reduction of elbow erosion peaks up to $31.4 \%$ can be achieved by placing the rib at $\theta=25^{\circ}$. Although the new structure elbow mentioned above can reduce the erosion of elbow, it is difficult to process the pipeline.

In this paper, the mechanical behavior of pipe during extrusion is fully considered, and the innovative shaped curved pipeline is established. The erosion characteristics of the innovative shaped curved pipeline is studied. The parameters of the innovative shaped curved pipeline are optimized from the depth of dent and the diameter of indenters. Finally, sensitivity analysis is carried out for different mass flow rate, particle diameter and flow velocity of the innovative shaped curved pipeline.

\section{Physical model}

Fig. 1, a show the normal curved pipeline studied in this paper. The diameter of the pipe is $D(D=3.5$ inches), and the bending radius $R=2 D$. In order to ensure that the fluid entering the elbow is in full development stage and to observe the particle trajectory after passing through the elbow, a straight tube of $6 D$ length is added upstream and downstream of the elbow. Fig. 1, b-d shows the manufacturing process of the innovative shaped curved pipeline. First, two cylindrical cylinders with a diameter of $D_{I}$ are placed at the distance from the entrance $3 D$. Then the indenter is used to squeeze the pipeline, leaving a dent with a depth of $H$ on the pipe. Finally, the flow field in the pipeline is extracted by Boolean operation. 


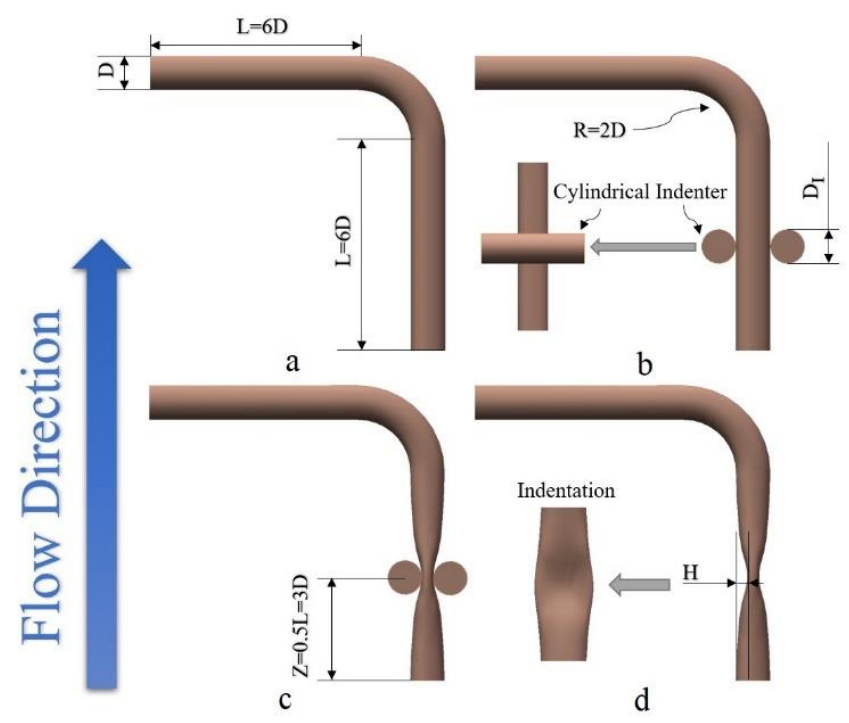

Fig. 1 Physical model

\section{Computation details}

\subsection{Governing equation}

1. Standard $K-\varepsilon$ turbulence model: Because the standard $K-\varepsilon$ turbulence model has the characteristics of high accuracy and simple application [13], the standard $K$ $\varepsilon$ turbulence model is used to study pipeline erosion. The standard turbulence model is described as follows:

$$
\begin{aligned}
& \rho \frac{D k}{D t}=\frac{\partial}{\partial x_{i}}\left[\left(\mu+\frac{\mu_{t}}{\sigma_{x}}\right) \frac{\partial k}{\partial x_{i}}\right]+G_{\mathrm{k}}+G_{b}-\rho \varepsilon-Y_{M}, \\
& \rho \frac{D \varepsilon}{D t}=\frac{\partial}{\partial x_{i}}\left[\left(\mu+\frac{\mu_{t}}{\sigma_{k}}\right) \frac{\partial \varepsilon}{\partial x_{i}}\right]+C_{1 \varepsilon} \frac{\varepsilon}{k}\left(G_{k}+C_{3 \varepsilon} G_{b}\right)- \\
& -C_{2 \varepsilon} \rho \frac{\varepsilon^{2}}{k},
\end{aligned}
$$

where: $G_{k}$ and $G_{b}$ are turbulent kinetic energy caused by average velocity gradient and buoyancy, respectively; $Y_{M}$ indicates the effect of turbulence pulsation on dissipation rate; $\mu_{t}$ is turbulent viscosity coefficient, $\mu_{t}=\rho C_{u} \frac{k^{2}}{\varepsilon} ;$ In FLUENT computational fluid dynamics software, $C_{1 \varepsilon}=1.44, C_{2 \varepsilon}=1.92, C_{\mu}=0.009$.

2. Particle motion trajectory model : Ignoring small forces such as force of flow field pressure gradient on particles, buoyancy, gravity, etc [14]. According to the calculated balance of forces acting on particles, the particle motion equation of particle motion trajectory in Cartesian coordinate system is obtained as follows:

$$
\frac{d u_{p}}{d t}=F_{D}\left(u-u_{p}\right)+\frac{g_{x}\left(\rho_{p}-\rho\right)}{\rho_{p}}+F_{x},
$$

where: $F_{D}\left(u-u_{p}\right)$ is the drag force per unit mass of particles, $F_{D}=\frac{18 \mu}{\rho_{\mathrm{p}} D_{p}^{2}} \cdot \frac{C_{D} R e}{24} ; u$ is the gas phase velocity; $u_{p}$ is the particle velocity; $\mu$ is the gas dynamic viscosity; $\rho$ is the gas density; $\rho_{p}$ is the particle density; $D_{p}$ is the particle diameter; $R e$ is the particle Reynolds number.

3. Collision model: There is energy loss in the process of solid particles impacting the pipeline, so its rebound speed is lower than the incident speed. The velocity distribution of solid particles before and after collision with the wall is shown in Fig. 2. Generally, the recovery coefficient (the ratio of rebound to incident velocity) can be used to describe this effect. The elastic recovery coefficient is represented by a normal component $e_{n}$ and a tangential component $e_{\tau}$, and its expression is as follows:

$$
e_{n}=\frac{u_{p 1}}{u_{p 2}}, e_{\tau}=\frac{v_{p 1}}{v_{p 2}},
$$

In the formula, $e_{n}$ and $e_{\tau}$ respectively represent the rate of change of momentum in the direction perpendicular to the wall and parallel to the wall (normal and tangential) before and after the particle colliding with the wall.

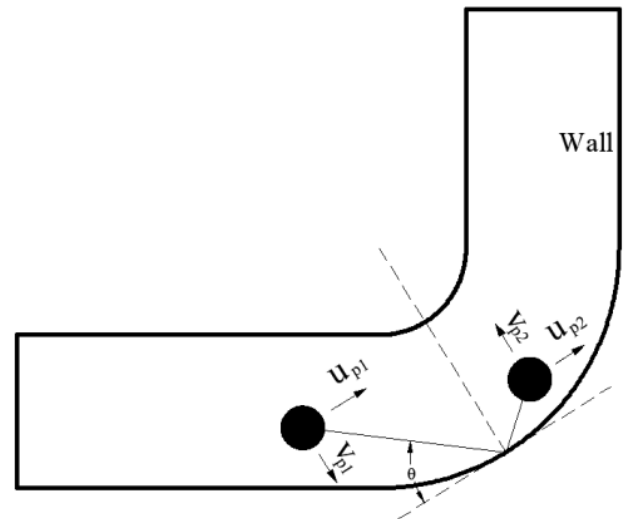

Fig. 2 Schematic diagram of particle collision with wall surface

Most of the materials used for manufacturing natural gas pipelines are carbon steel. Referring to FLUENT's help document, the following recovery coefficient formulas are given for the recovery of normal and tangential recovery coefficients in the calculation [15]:

$$
\begin{aligned}
& e_{n}=0.993-0.0307 \theta+0.000475 \theta^{2}-0.00000261 \theta^{3} \\
& e_{\tau}=0.988-0.028 \theta+0.000642 \theta^{2}-0.00000356 \theta^{3},
\end{aligned}
$$

The impact angle function uses a piecewise linear function and sets the function values at $0^{\circ}, 20^{\circ}, 30^{\circ}, 45^{\circ}$ and $90^{\circ}$ to $0,0.8,1,0.5$ and 0.4 , respectively. In this paper, the particle diameter function adopts the constant of $1.8 \mathrm{e}-9$ and the velocity exponent function of 2.6.

4. Erosion model: This paper uses the particle erosion and accretion theory provided by FLUENT to calculate the erosion of solid particles on natural gas pipelines. The general form of the predicted erosion model in FLUENT is as follows:

$$
R_{\text {erosion }}=\sum_{p=1}^{N_{\text {particles }}} \frac{m_{p} C\left(d_{p}\right) f(\alpha) v^{b(v)}}{A_{\text {face }}},
$$


where: $\dot{m}_{p}$ is the mass flow rate of particles; $C\left(d_{p}\right)$ is a function of particle diameter; $\alpha$ is the impact angle formed between the incident direction of particles and the wall surface; $f(\alpha)$ is a function of the impact angle; $v$ is the relative velocity of particles; $b(v)$ is a function of the relative velocity of particles; $A_{\text {face }}$ is the surface area of the wall unit.

5. Basic Control Equations: The conservation law of physics is the basic law that all fluids must follow when they flow. In all the processes of flow, it can be explained by the conservation of mass, momentum and energy. The mathematical differential expressions of the three basic governing equations are as follows.

Continuity equation:

$$
\frac{\partial}{\partial x_{i}}\left(\rho u_{i}\right)=0
$$

Momentum equation:

$$
\frac{\partial}{\partial X_{i}}\left(\rho u_{i} u_{j}\right)=-\frac{\partial p}{\partial X_{i}}+\frac{\partial}{\partial X_{j}}\left(\mu+\mu_{t}\right)\left(\frac{\partial u_{i}}{\partial X_{j}}+\frac{\partial u_{j}}{\partial X_{i}}\right)
$$

Energy equation:

$$
\frac{\partial}{\partial X_{i}}\left(u_{i} T\right)=\frac{\partial}{\partial X_{i}}\left[\left(\frac{\mu}{\operatorname{Pr}}+\frac{\mu_{t}}{\operatorname{Pr}_{t}}\right) \frac{\partial T}{\partial X_{i}}\right]
$$

Turbulence kinetic energy $(k)$ equation:

$$
\frac{\partial}{\partial X_{j}}\left(\rho k u_{j}\right)=\frac{\partial}{\partial X_{j}}\left[\left(\mu+\frac{\mu_{t}}{\sigma_{k}}\right) \frac{\partial k}{\partial X_{j}}\right]+\Gamma-\rho \varepsilon .
$$

Specific dissipation rate $(\varepsilon)$ equation:

$$
\begin{aligned}
& \frac{\partial}{\partial X_{j}}\left(\rho \varepsilon u_{j}\right)=\frac{\partial}{\partial X_{j}}\left[\left(\mu+\frac{\mu_{t}}{\omega_{\varepsilon}}\right) \frac{\partial \varepsilon}{\partial X_{j}}\right]+C_{1} \Gamma \varepsilon- \\
& -C_{2} \frac{\varepsilon^{2}}{k+\sqrt{v \varepsilon}} .
\end{aligned}
$$

Where $\Gamma$ can be expressed as follows:

$$
\begin{aligned}
& \Gamma=-\overline{u_{i} u_{j}} \frac{\partial u_{i}}{\partial u_{j}}=\frac{\mu_{t}}{\rho}\left(\frac{\partial u_{i}}{\partial X_{j}}+\frac{\partial u_{j}}{\partial X_{i}}\right) \frac{\partial u_{i}}{\partial X_{j}}, \\
& C_{1}=\operatorname{Max}\left[0.43 \frac{\mu_{t}}{\mu_{t}+5}\right], C_{2}=1, \\
& \sigma_{k}=1, \sigma_{\varepsilon}=1.2, \mu_{t}=\rho C_{u} \frac{k^{2}}{\varepsilon}
\end{aligned}
$$

where: $\rho$ is fluid density; $u$ is velocity; $p$ is pressure; $T$ is temperature; $\mu_{t}$ is turbulent viscosity; $\mu$ is dynamic viscosity; $\operatorname{Pr}$ is the Prandtl number.
6. Data reduction: A PEC (performance evaluation criteria) considering both the maximum erosion rate of the normal curved pipeline and the maximum erosion rate of innovative shaped curved pipeline, which is defined as follows:

$$
P E C=\frac{E_{i}-E_{n}}{E_{n}},
$$

where: $E_{i}$ is the maximum erosion rate of innovative shaped curved pipeline; $E_{n}$ is the maximum erosion rate of the normal curved pipeline.

\subsection{Grid details and independent test}

The mesh is divided as shown in Figure 3. Because the erosion at elbow is more severe, the mesh is encrypted at elbow. The numerical simulation is carried out by using the models with 130042, 320464, 403210, 732012 and 1020135 meshes. As shown in Figure 4, when the number of grids reaches 403210, the increase of the number of grids has little effect on the maximum wear rate, so it is considered that the requirement of grid independence can be met.

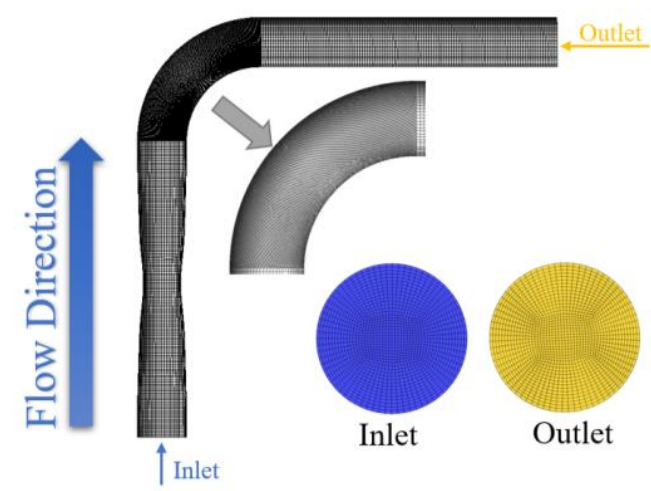

Fig. 3 Grid details

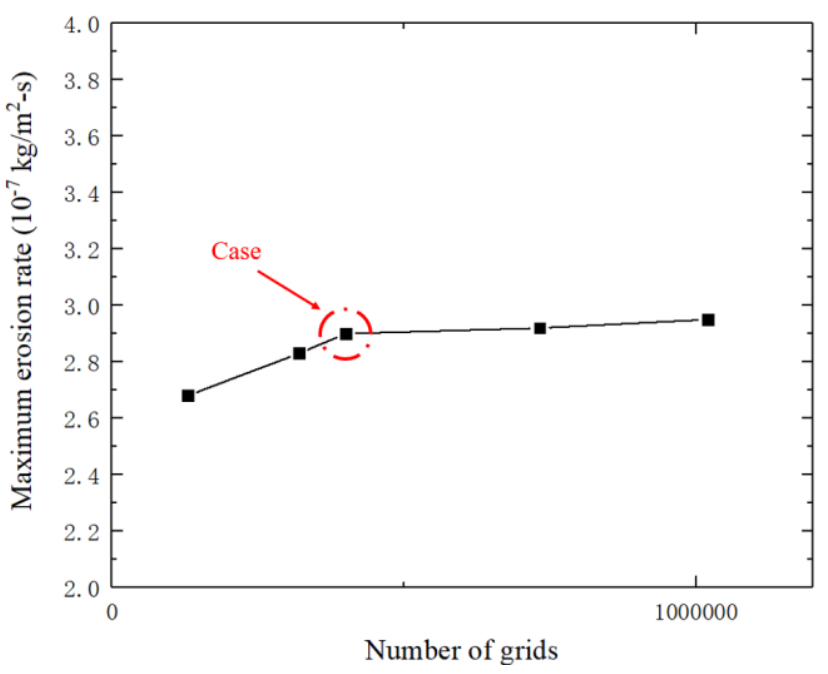

Fig. 4 Independent test

\section{Results and discussions}

\subsection{Effects of indentation depth}

Fig. 5 shows the distribution of erosion rate with different dent depths when particle diameter is $0.0002 \mathrm{~m}$, 
mass flow is $0.002 \mathrm{~kg} / \mathrm{s}$, particle velocity is $10 \mathrm{~m} / \mathrm{s}$ and indenter diameter is $D_{I}=D=3.5$-inch. It can be seen from Fig. 5 that the erosion mainly occurs at the elbow. The main erosion area is approximately elliptical. At the same time, in the area near the downstream exit of the elbow, two long strip erosion areas (similar to the "V" shape) are formed. As the dent depth increases from 0.25 inches to $1 \mathrm{inch}$, the core of erosion area decreases significantly, and the shape of the main erosion area gradually develops from ellipse to circle. At the same time, with the increase of dent depth, there will be a new phenomenon of erosion in the dent. Because the number of particles in the impact dent is obviously smaller than that in the impact elbow, the erosion in the dent is not serious at the elbow.

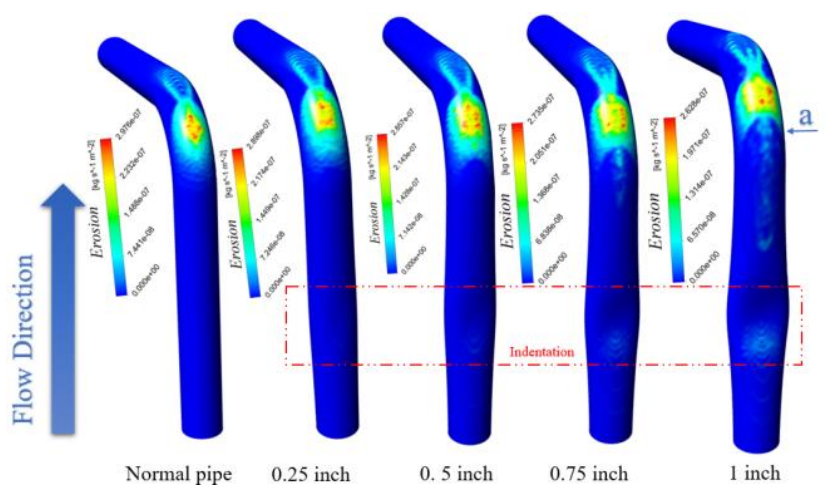

Fig. 5 Erosion distribution of pipeline under different depth of indentation

Figs. 6 and 7 show the velocity and pressure distribution of pipe flow field at different dent depths when particle diameter is $0.0002 \mathrm{~m}$, mass flow rate is $0.002 \mathrm{~kg} / \mathrm{s}$, particle velocity is $10 \mathrm{~m} / \mathrm{s}$ and indenter diameter is $D_{I}=D=$ 3.5-inch, respectively. Generally speaking, when the dent does not exist, the flow field in the straight pipe section at the elbow entrance is relatively stable, and the pressure of the flow field decreases at a constant speed in the direction of flow. Because of the effect of viscous force, the velocity distribution of the fluid is parabolic, that is, the velocity near the wall is smaller, and the velocity at the central axis of the pipeline is larger. When the fluid passes through the elbow, because the flow direction changes dramatically, the law of steady change of velocity and pressure field is destroyed, resulting in high pressure on the outside of the elbow section, but low flow velocity, and low pressure ("a" area) on the inside of the elbow tube, but high flow velocity. When the fluid flows out of the elbow, the flow direction of the fluid gradually stabilizes, so the fluid restores to the fluid state of the straight-line section in front of the elbow. When the dent exists, the cross-sectional area of the pipeline suddenly decreases, resulting in a sudden increase in flow velocity and a sudden decrease in pressure at the dent. Similarly, after the fluid flows through the dent area, the fluid state gradually restores to the state before entering the dent area, so the fluid state flowing through the elbow is almost the same as when the dent does not exist.

Fig. 8 shows the trajectory of particle movement in the curved pipeline with dent and normal curved pipeline. In the normal curved pipeline, particles move smoothly and hardly collide in the straight pipe section. At the elbow, most particles collide with the wall in area A, so the most serious area of erosion occurs in area A. At the same time, particles cross at place B after collision. The particles on the right side move to the left side, while the particles on the left side move to the right side, thus forming a "V" erosion area. In curved pipeline with indentation, the particle trajectory will deflect when it impacts on the wall of indentation, resulting in the decrease of the particle number at " $\mathrm{A}$ " area, so the erosion area will change. At the same time, the energy loss and particle motion dispersion will occur after the particle impacts on the wall of indentation, resulting in the decrease of the erosion rate at the elbow and the dispersion of the erosion area.

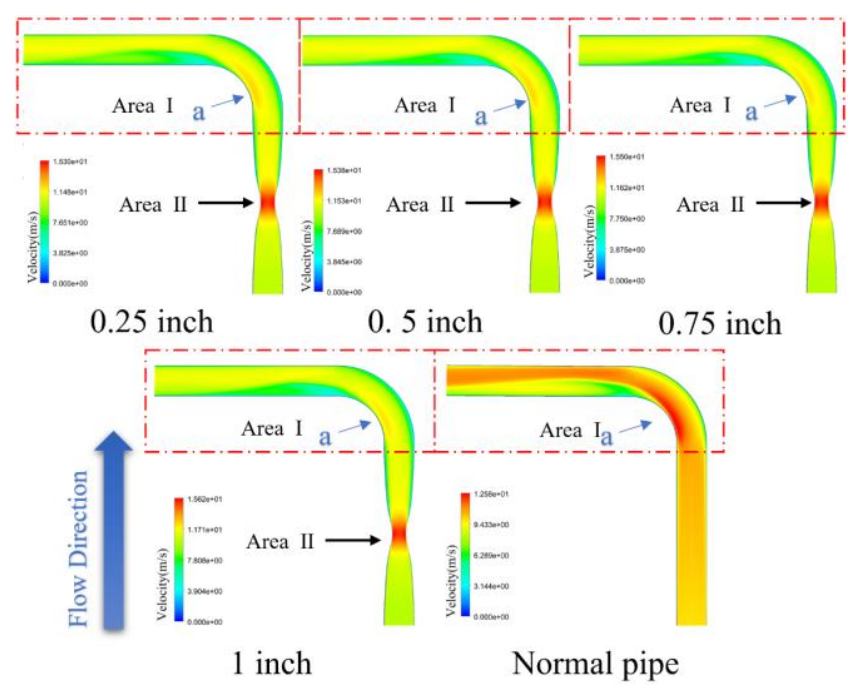

Fig. 6 Velocity distribution of flow field under different of indentation

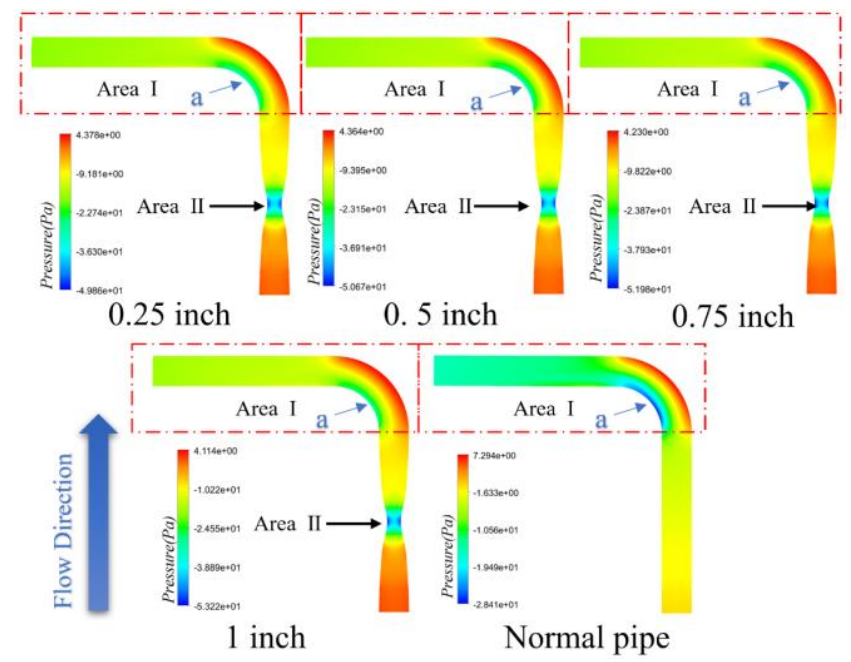

Fig. 7 Pressure distribution of flow field under different of indentation

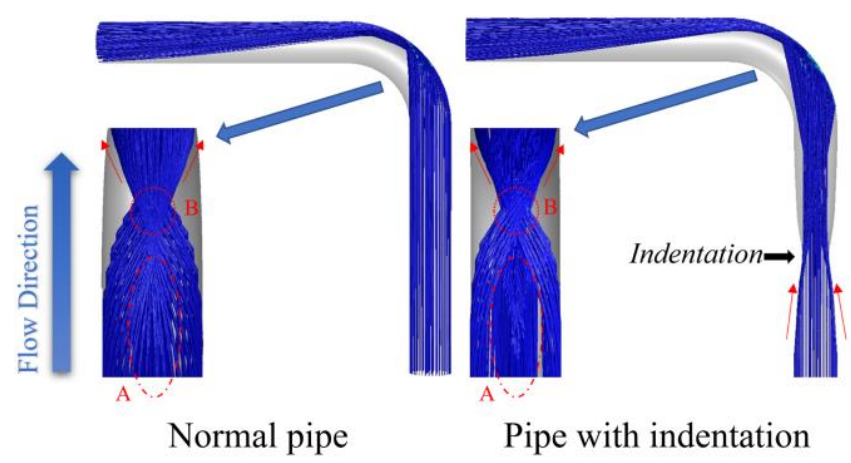

Fig. 8 Trajectories of particles in two kinds of pipes 
The maximum erosion rate is an important parameter for predicting the failure of pipeline. In Figure 9, it can be seen that the existence of indentation can reduce the maximum erosion rate. The more the indentation depth increases, the more obvious the reduction effect is. When the indentation depth is 1 inch, the PEC value reaches 0.13 , indicating that the maximum erosion wear rate decreases by $13 \%$.

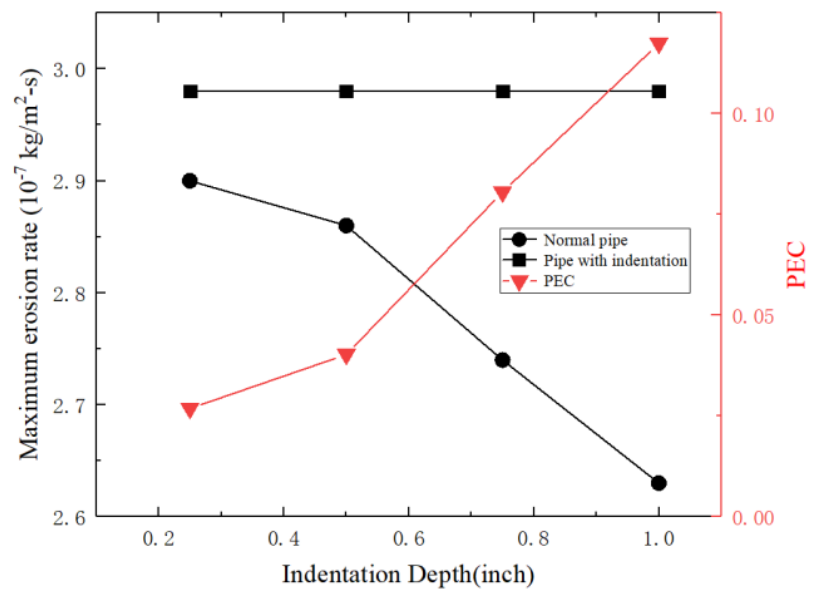

Fig. 9 Maximum erosion rate and PEC variation at different depths of indentation

\subsection{Effects of indenter diameter}

Fig. 10 shows the distribution of erosion rate with particle diameter of $0.0002 \mathrm{~m}$, mass flow rate of $0.002 \mathrm{~kg} / \mathrm{s}$, particle velocity of $10 \mathrm{~m} / \mathrm{s}$, pit depth of 1 inch and different indenter diameter of $D_{I}$. As can be seen from Fig. 10, the erosion area on the pipeline with the different indentation depth is roughly the same. However, when the diameter of indenter increases, the shape of the indentation will become flat, which will weaken the effect of the indentation on the particle trajectory change, and cause a large number of particles to impact the wall in area A, resulting in the local maximum erosion rate. Fig. 11 shows that the maximum erosion rate increases linearly with the increase of indenter diameter, but the maximum erosion rate of the innovative shaped curved pipeline is still lower than that of normal curved pipeline. When the diameter of indenter reaches 6.5 inches, PEC is only 0.04 , which hardly reduces the maximum erosion rate. Therefore, the choice of indenter diameter should not be too large.

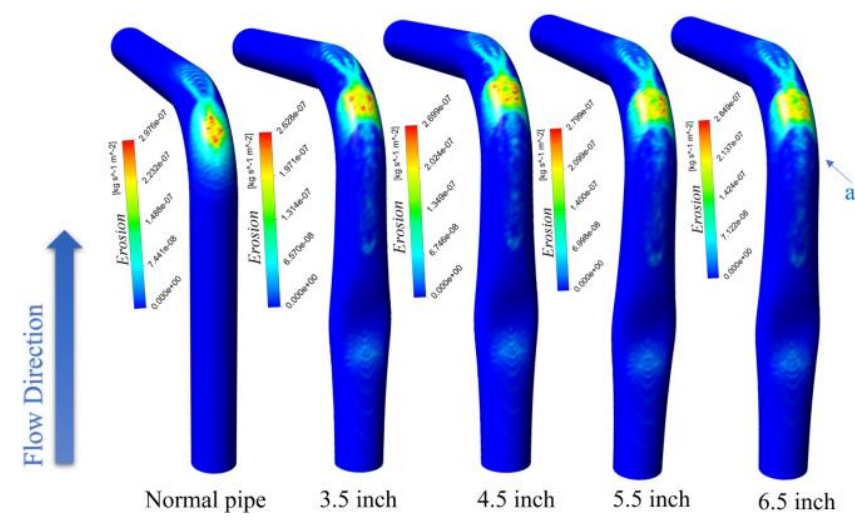

Fig. 10 Erosion distribution of pipeline under different diameter of indenter

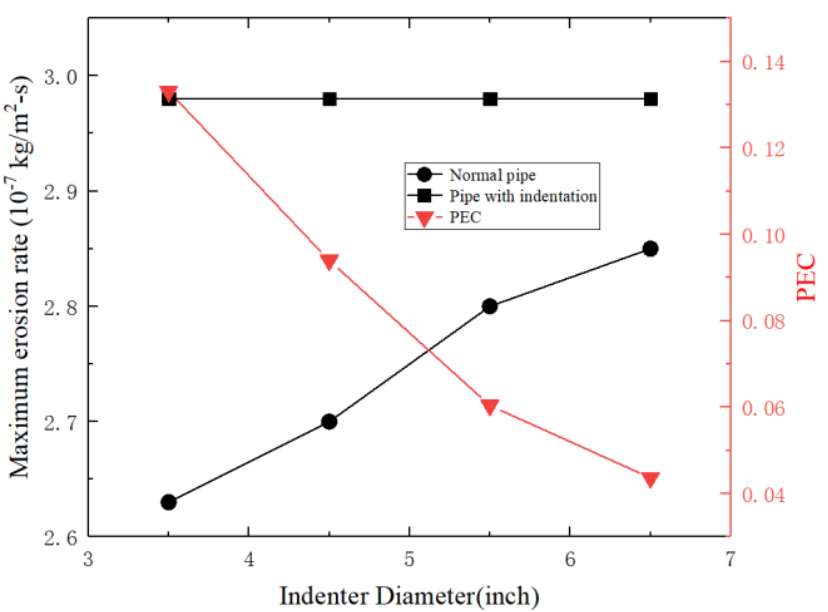

Fig. 11 Maximum erosion rate and PEC variation under different diameter of indenter

\subsection{Effects of particle parameters}

Figure 12 shows the distribution of erosion rate with particle diameter of $0.0002 \mathrm{~m}$, indenter diameter is 3.5 inch, particle velocity of $10 \mathrm{~m} / \mathrm{s}$, pit depth is 1 inch and different mass flow rate. Figure 13 shows the distribution of erosion rate with indenter diameter is 3.5 -inch, mass flow rate of $0.002 \mathrm{~kg} / \mathrm{s}$, particle velocity of $10 \mathrm{~m} / \mathrm{s}$, pit depth is 1 inch and different particle diameter. Figure 14 shows the distribution of erosion rate with particle diameter is $0.0002 \mathrm{~m}$, indenter diameter is 3.5 -inch, mass flow rate of $0.002 \mathrm{~kg} / \mathrm{s}$, pit depth is 1 inch and different particle velocity. It can be seen that when the particle parameters (mass flow rate, diameter and velocity) change, the distribution of erosion in area "a" at the pit and area B at the elbow is almost unchanged, which indicates that the particle parameters have no effect on the distribution of erosion zone on the innovative shaped curved pipeline.

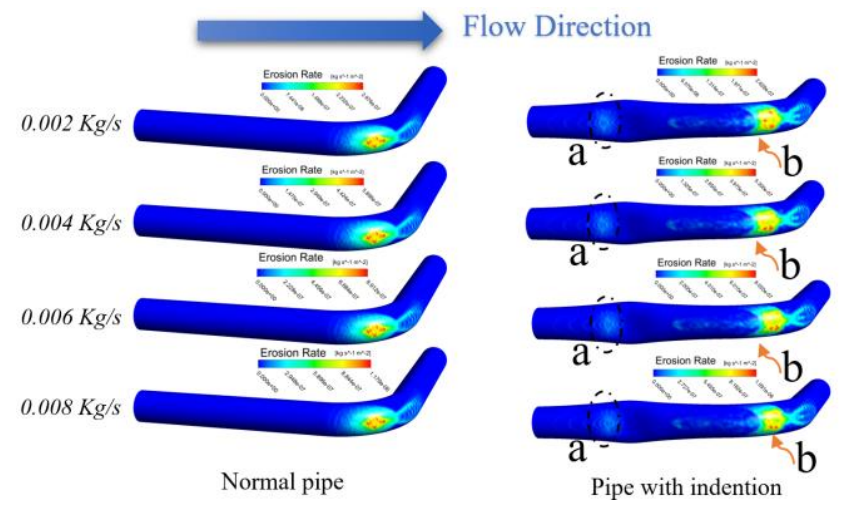

Fig. 12 Erosion distribution of pipeline under different mass flow rate

Fig. 15 shows the maximum erosion rate and PEC when particle diameter of $0.0002 \mathrm{~m}$, indenter diameter is 3.5 -inch, particle velocity of $10 \mathrm{~m} / \mathrm{s}$, pit depth is $1 \mathrm{inch}$ and different mass flow rate. Figure 16 shows the maximum erosion rate and PEC when indenter diameter is 3.5-inch, mass flow rate of $0.002 \mathrm{~kg} / \mathrm{s}$, particle velocity of $10 \mathrm{~m} / \mathrm{s}$, pit depth is 1 inch and different particle diameter. Figure 17 shows the maximum erosion rate and PEC when particle diameter is $0.0002 \mathrm{~m}$, indenter diameter is 3.5 -inch, mass flow rate of $0.002 \mathrm{~kg} / \mathrm{s}$, pit depth is 1 inch and different particle velocity. It can be seen that when the particle parameters 
(mass flow rate, diameter and velocity) change, the erosion rate of normal curved pipeline and innovative shaped curved pipeline both changes significantly, which indicates that the particle parameters are the parameters affecting the erosion rate.

Fig. 15 shows that with the increase of mass flow rate, the maximum erosion rate of normal curved pipeline and innovative shaped curved pipeline both increases, while the PEC value decreases slowly at first and then rapidly. When the mass flow rate of particles is large, the effect of indentation on the reduction of maximum erosion rate decreases. Therefore, to achieve a good optimization effect, the mass flow rate of particles needs to be controlled.

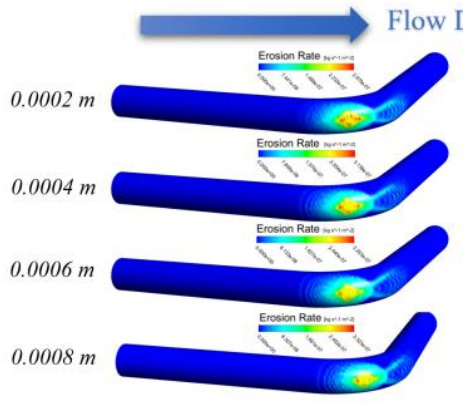

Normal pipe

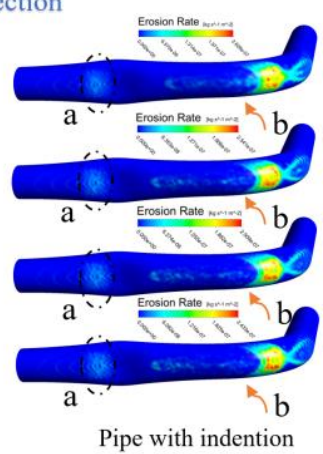

Fig. 13 Erosion distribution of pipeline under different particle diameter

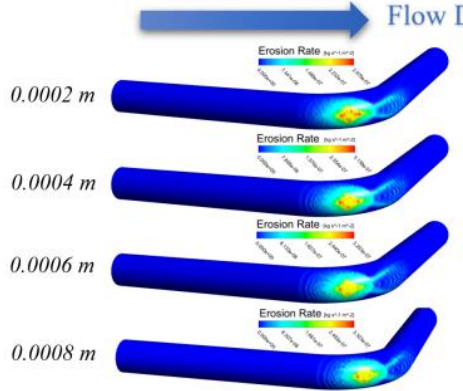

Normal pipe

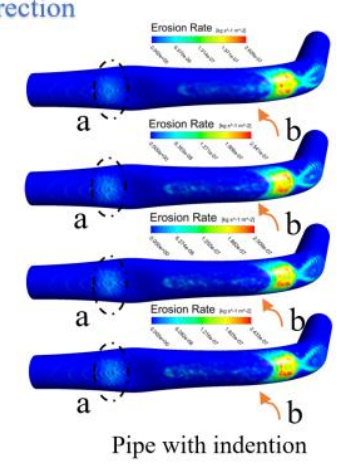

Fig. 14 Erosion distribution of pipeline under different particle velocity

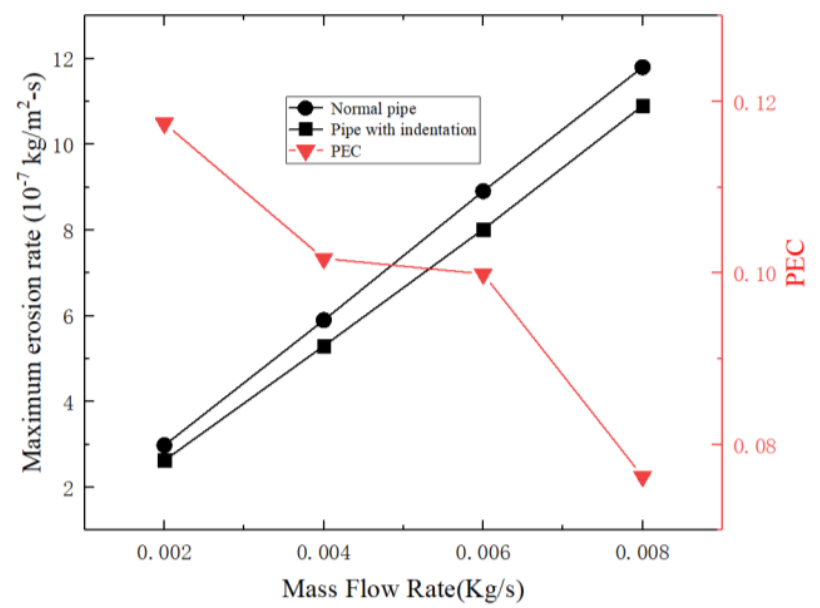

Fig. 15 Maximum erosion rate and PEC variation under different mass flow rates

With the increase of particle diameter, the probability of particle impacting on the wall at the indentation will be increased, so more particles will be impacting on the wall at the indentation, resulting in the change of particle trajectory and energy loss. Fig. 16 shows that with the increase of particle diameter, the maximum erosion rate of normal curved pipeline increases, while the maximum erosion rate of innovative shaped curved pipeline decreases, and the PEC increases almost linearly. Therefore, when the diameter of particles in natural gas pipeline is large, the bend with pits can effectively reduce the maximum erosion wear rate.

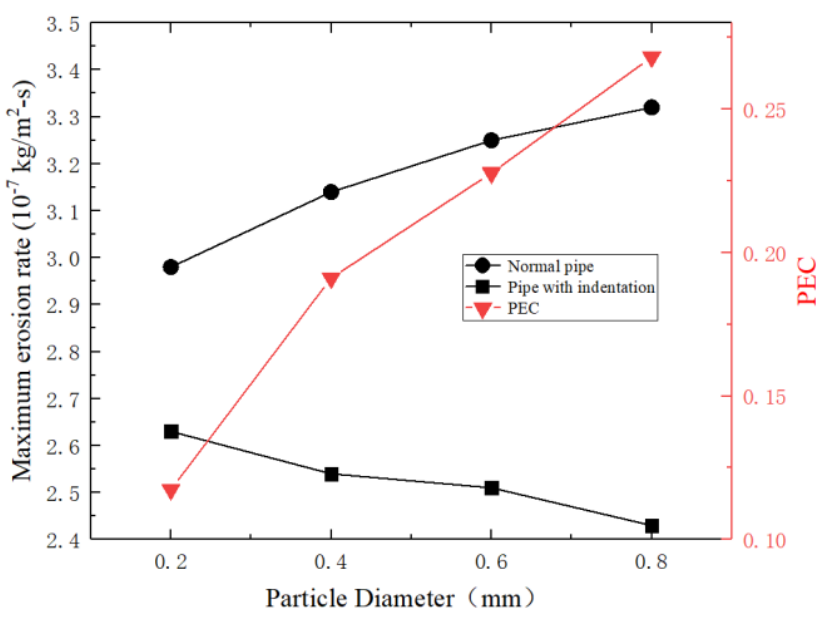

Fig. 16 Maximum erosion rate and PEC variation under different particle diameters

Fig. 17 shows that with the increase of particle velocity, the maximum erosion rate of normal curved pipeline and innovative shaped curved pipeline both increases, but PEC increases rapidly at first and then tends to be stable. Therefore, when the particle velocity is high, the innovative shaped curved pipeline can also effectively lower the maximum erosion rate.

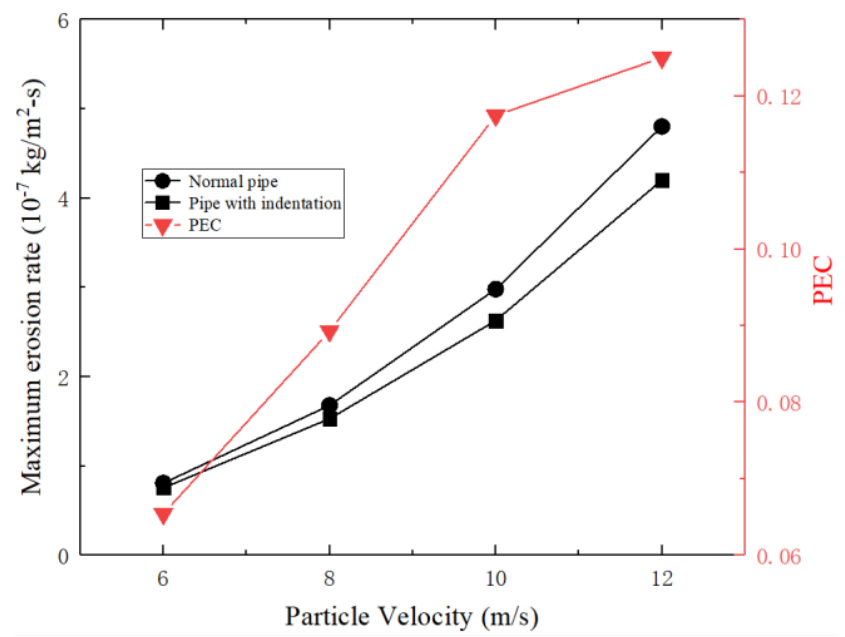

Fig. 17 Maximum erosion rate and PEC variation under different particle velocities

\section{Conclusions}

In this paper, an innovative shaped curved pipeline is presented. The numerical simulation of erosion mechanism is carried out from two aspects of indentation parameters and particle parameters. The results show that:

1.Because the existence of indentation can disperse the area where particles impact the wall at the elbow and 
reduce the energy of particles after the impact, the innovative shaped curved pipeline can reduce the maximum erosion rate in the elbow. In the working conditions studied in this paper, the maximum PEC can reach 0.268 , which means that the maximum erosion rate can be reduced by $26.8 \%$.

2.Within a certain range, the ability to reduce the maximum erosion rate increases with the increase of indentation depth. With the increase of indenter diameter, the ability to reduce the maximum erosion rate decreases.

3.Particle parameters play an important role in erosion rate. In the case of larger particle mass flow rate and particle velocity, the indentation has better effect on reducing the maximum erosion rate of elbow.

\section{References}

1. Tilly, G P. 1979. Erosion caused by impact of solid particles, Treatise on Materials Science \& Technology 13: 287-319. http://dx.doi.org/10.1016/S0161-9160(13)70071-1.

2. Finnie, I. 1958. The mechanism of erosion of ductile metals, 3rd U.S. Nat. congress of Applied Mechanics, ASME, New York, 527-532. http://dx.doi.org/10.1016/0043-1648(60)90193-9.

3. Finnie, I. 1960. Erosion of surfaces by solid particles, Wear 3(2):87-103. https://doi.org/10.1016/0043-1648(60)90055-7.

4. Salik, J.; Buckey, D.; Brainard, W. 1981. The effect of mechanical surface and heat treatments on erosion resistance of 6061 aluminum alloy, Wear 65: 351-358. https://doi.org/10.1016/0043-1648(81)90061-2.

5. Mclaury, B. S.1996. Predicting Solid Particle Erosion Resulting from Turbulent Fluctuations in Oilfield Geometries, University of Tulsa, PhD Thesis. https://elibrary.ru/item.asp?id=5421903.

6. Chen, J.; Wang, Y.; Li, X.; He, R.; Han, S.; Chen, Y. 2015. Reprint of "erosion prediction of liquid-particle two-phase flow in pipeline elbows via cfd-dem coupling method", Powder Technology 282: 25-31. https://doi.org/10.1016/j.powtec.2015.05.037.

7. Peng, W.; Cao, X. 2016. Numerical prediction of erosion distributions and solid particle trajectories in elbows for gas-solid flow, Journal of Natural Gas Science and Engineering 30: 455-470. https://doi.org/10.1016/j.jngse.2016.02.008.

8. Peng, W.; Cao, X. 2016. Numerical simulation of solid particle erosion in pipe bends for liquid-solid flow, Powder technology 294: 266-279. https://doi.org/10.1016/j.powtec.2016.02.030.

9. dos Santos, V. F.; de Souza, F. J.; Duarte, C. A. R. 2016. Reducing bend erosion with a twisted tape insert, Powder Technology 301: 889-910. https://doi.org/10.1016/j.powtec.2016.07.020.

10. Lain, S.; Sommerfeld, M. 2019. Numerical prediction of particle erosion of pipe bends, Advanced Powder Technology 30(2): 366-383. https://doi.org/10.1016/j.apt.2018.11.014.

11. Duarte, C. A. R.; de Souza, F. J. 2017. Innovative pipe wall design to mitigate elbow erosion: A CFD analysis, Wear 380: 176-190

https://doi.org/10.1016/j.wear.2017.03.015.

12. Zhu, H. J.; Li, S. 2018. Numerical analysis of mitigating elbow erosion with a rib, Powder technology 330: 445-460. https://doi.org/10.1016/j.powtec.2018.02.046.

13. Launder, B. E.; Spalding, D. B. 1974. The numerical computation of turbulent flows, Comput. Methods Appl. Mech. Eng. 3(2): 269-289. https://doi.org/10.1016/0045-7825(74)90029-2.

14. Badr, H. M.; Habib, M. A.; Ben-Mansour, R.; Said, S. A. M. 2005. Numerical investigation of erosion threshold velocity in a pipe with sudden contraction, Comput. Fluids 34(6): 721-742.

https://doi.org/10.1016/j.compfluid.2004.05.010.

15. Zhang, J.; Yi, H.; Huang, Z.; Du, J. 2019. Erosion mechanism and sensitivity parameter analysis of natural gas curved pipeline, Journal of Pressure Vessel Technology 141(3): 034502. http://dx.doi.org/10.1115/1.4043011.

\section{Mo, Z. Wang, J. Du, H. Yi, J. Tang}

\section{EROSION MECHANISM AND SENSITIVITY PARAMETER ANALYSIS OF AN INNOVATIVE SHAPED CURVED PIPELINE}

\section{S u m m a r y}

In the process of natural gas transportation, it is unavoidable for particles to collide with the wall, which will cause erosion of curved pipeline. Reasonable curved pipeline structure can effectively avoid the erosion failure. In this paper, an innovative shaped curved pipeline formed by extrusion of cylindrical indenter is presented. The erosion mechanism and sensitivity parameter analysis of the innovative shaped curved pipeline is studied by numerical simulation and compared with that of ordinary elbow. In addition, the effects of extrusion parameters and particle parameters on erosion of innovative shaped curved pipeline were also studied. The results show that the dent can effectively reduce the maximum erosion rate of elbow. With the increase of dent depth, the maximum erosion rate of elbow is decreasing. With the increase of indenter diameter, the ability to reduce the maximum erosion rate decreases. Under the harsh conditions of large particle diameter and high particle velocity, the dent has a better ability to reduce the maximum erosion wear rate, and the maximum erosion rate can be reduced by $26.8 \%$.

Keywords: innovative shaped tube, gas/solid two-phase flow, erosion, numerical simulation.

Received February 09, 2020

Accepted December 01, 2020

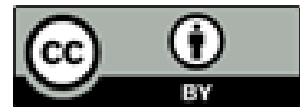

This article is an Open Access article distributed under the terms and conditions of the Creative Commons Attribution 4.0 (CC BY 4.0) License (http://creativecommons.org/licenses/by/4.0/). 